\title{
Performance of an Energy compensated optical Tomographic Atom Probe (EcoTAP)
}

\section{Renaud}

CAMECA, 103 Bd. St-Denis, 92403, Courbevoie cedex, France.

The $3 \mathrm{D}$ atom probe is a quantitative measuring instrument, on a nearly atomic scale [1]. This instrument enables the 3D reconstruction of a small volume of matter probed (typically $10 \mathrm{~mm} \times 10$ $\mathrm{mm} \times 50 \mathrm{Nm}$ ) of a metallic material (Figure 1) [2]. The sample is prepared in a form of a very sharp tip. Under the effect of high-voltage pulses, the atoms of surface are evaporated by field effect and projected on a position sensitive detector. This detector allows the localization of the impacts in synchronism with the measurement of times of flight.

The GPM at Rouen University (France) has developed a new 3D atom probe : the EcoTAP. In this presentation, we propose to present this new instrument commercialized by Cameca. Some results obtained with the EcoTAP will also be presented in order to evaluate the contribution of the instrument in materials science..

This instrument presents to major advantages :

- The detection system that allows quantitative measurement.

- The energy compensation device

The instrument is equipped with a position sensitive detector that allows on a same pulse the detection of some ions (0 to 10) [3]. These ions can strike the detector at the same time or with times of flight different from a few nanoseconds. For each detected ion, position and time of flight are accurately determined. This detector consists of a CCD camera associated with a MCP phosphor screen assembly (Figure 2). Due to the MCP, all the ions are not detected. For the evaluation of the performances of the instrument, it has been very important to quantify the detection efficiency of the detector. For this purpose a new way of accurately measuring the absolute detection efficiency has been done and will be exposed [4].

To improve the mass resolution, the EcoTAP is equipped with an energy compensation device. Because of energy deficits of ions, the mass resolution of a straight 3D atom-probe is rather poor. The energy deficits have been corrected by incorporating a reflectron lens to the instrument. We have compared the mass resolution of a straight atom probe with the mass resolution of the EcoTAP. The results of this comparison (Figure 3) clearly indicate that with the reflectron lens the mass resolution has been increased by a factor of 2.5 .

[1] D. Blavette et al., Nature 363 (1993) 432 - 434

[2] X. Sauvage et al., Acta mater. vol 49-01 Issues 3 (2001) 389-394

[3] L. Renaud et al., Nuclear Instruments and Methods in Physics Research Section A: Vo 477, Issues 1-3, 21 January 2002, 150-154

[4] B. Deconihout et al., Review of Scientific Instruments, vol. 73-4, April 2002, 1734-1740 


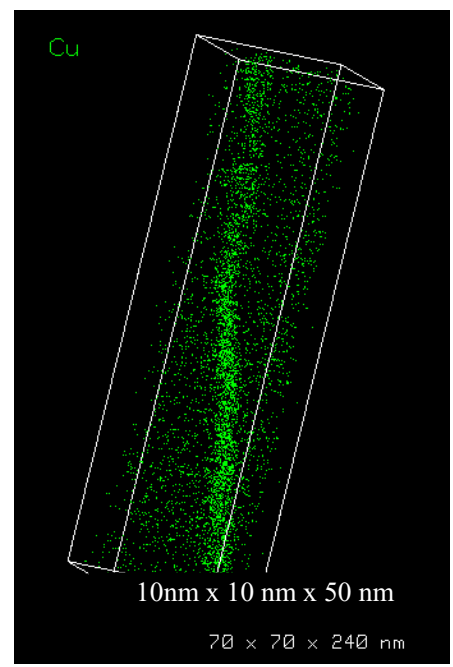

Figure $1: 3 \mathrm{D}$ image obtained from the analysis of a $\mathrm{CuNb}$ alloy. Each dot is a $\mathrm{Cu}$ atom. This distribution shows the presence of a thin wire of $\mathrm{Cu}(2 \mathrm{~nm}$ diameter $)$ in the material.

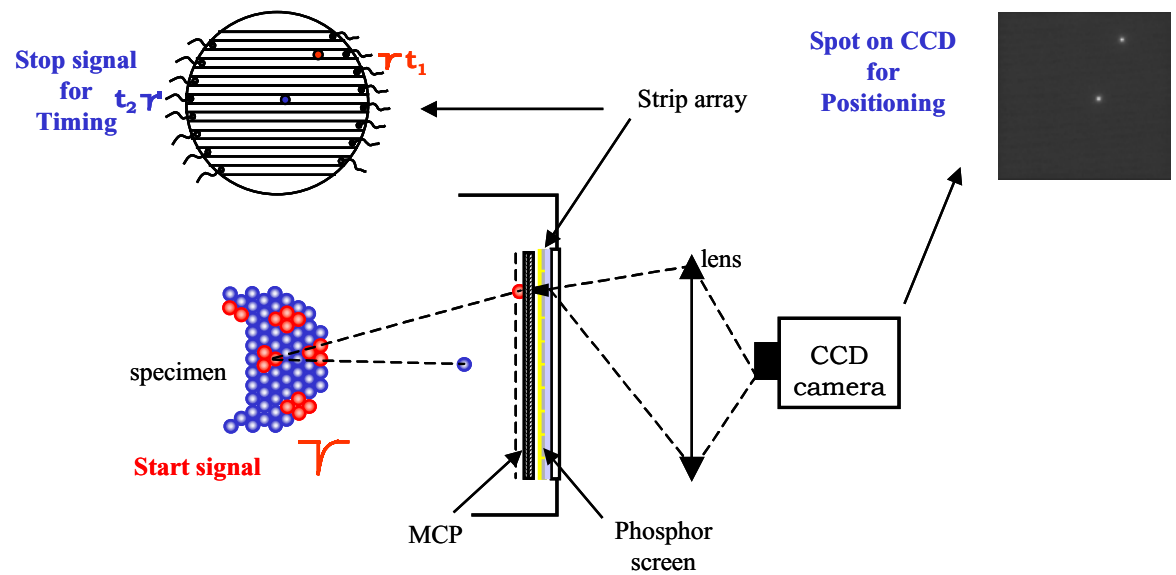

Figure 2 : Principle of the 3D atom probe and design the position sensitive detector.
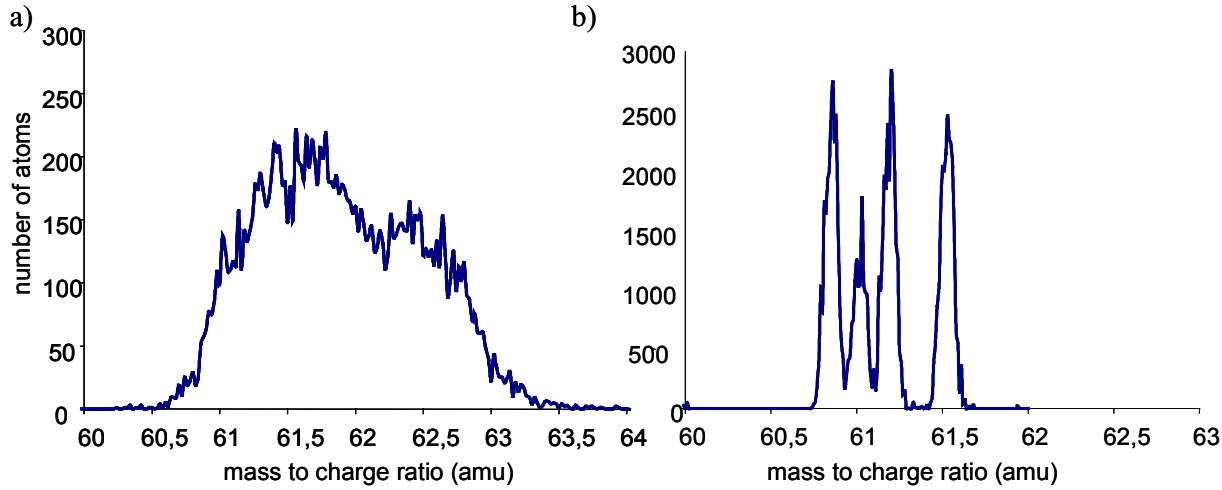

Figure 3 : Mass spectra obtained on a tungsten alloy. The image a) is obtained with a straight atom probe and the image $b$ ) is obtained with the EcoTAP. 